\title{
Diseño y desarrollo de escenarios de simulación clínica: análisis de cursos para el entrenamiento de anestesiólogos
}

José M. Maestre, Rodrigo Sancho, José L. Rábago, Alejandro Martínez, Elena Rojo, Ignacio del Moral

Introducción. La simulación se está integrando en los programas docentes de los residentes y en la formación continuada de los especialistas. El objetivo de este artículo es identificar y estandarizar las mejores prácticas para diseñar e implementar un escenario de simulación clínica.

Materiales y métodos. Se analizan los cursos realizados para la especialidad de Anestesiología y Reanimación en el Hospital Virtual Valdecilla durante el período septiembre 2008-junio 2011.

Resultados. Se revisan un total de 104 escenarios correspondientes a 14 cursos de formación continuada de médicos especialistas, y 13 realizados para el programa de integración de la simulación clínica en el programa formativo de los residentes. Se identifican y estandarizan las fases seguidas para el diseño, puesta en marcha y posterior refinamiento de cada escenario, así como los criterios utilizados para la toma de decisiones en cada una de ellas.

Conclusiones. En nuestra experiencia, el análisis correcto de los objetivos docentes, y que éstos sean la guía para el diseño de un escenario clínico, es el factor individual más importante para el éxito de un programa de simulación.

Palabras clave. Anestesiología. Aprendizaje experiencial. Diseño de escenarios. Entrenamiento. Reanimación. Simulación clínica.

\section{Design and development of clinical simulation scenarios: analysis of courses for anesthesiologists}

Introduction. Simulation is integrating into the residency programs and for continuing medical education (CME). The aim of this article is identify and standardize the best practices to design and develop clinical simulation scenarios for training exercises.

Materials and methods. We analyze the courses for Anesthesia and Critical Care performed in Valdecilla Virtual Hospital from September 2008 to June 2011.

Results. A total of 104 scenarios were reviewed, related to 14 CME and 13 resident courses. The resultant phases followed to design, build and refine a clinical simulation scenario, and the criteria utilized to make the decisions in each step are presented.

Conclusions. In our opinion clearly define the training objectives is the most important single factor to guide the scenario design and determine a meaningful simulation experience.

Key words. Anesthesia. Critical care. Experiential learning. Scenario design. Simulation. Training.

\section{Introducción}

La simulación clínica se está incorporando progresivamente tanto a los programas docentes de los residentes de anestesiología como a la formación continuada de los especialistas, en respuesta a una asistencia sanitaria cada vez más compleja $[1,2]$. Ello se debe a varios motivos. Por un lado, es una técnica pedagógica que permite el entrenamiento en un entorno realista y seguro, sin poner en riesgo a pacientes y profesionales [3]. Por otro, se ha demostrado efectiva para adquirir habilidades técnicas e integrar conocimientos y habilidades clínicas complejas, aumentado el grado de retención de lo aprendido cuando se compara con los métodos docentes tradicionales [4]. Además, tras su implementación, se ha evidenciado que mejora el cuidado de los pacientes y disminuye las complicaciones clínicas [5].

En este entorno, numerosos centros e instituciones docentes en nuestro sistema de salud están adquiriendo equipos de simulación de diferente grado de fidelidad, favorecido también por el coste actual más asequible de la tecnología. Cada centro los utiliza en diversos ambientes clínicos, con múltiples
Hospital Virtual Valdecilla. Santander, Cantabria, España.

Correspondencia: Dr. José María Maestre. Hospital Virtual Valdecilla. Avda. Valdecilla, $s / n$ E-39008 Santander (Cantabria). Fax: 34942203853 . E-mail: jmmaestre@hvvaldecilla.es

Agradecimientos: A los miembros del Servicio de Anestesiología y Reanimación del Hospital Universitario Marqués de Valdecilla, por su constante apoyo para la realización del programa de simulación.

Conflicto de intereses: No declarado.

Conflict of interests: None declared.

(c) 2013 FEM 
tipos de pacientes, y por participantes de distinta experiencia [6,7]. A su vez, se han descrito criterios y recomendaciones para identificar las áreas y competencias que más se beneficien de este tipo de entrenamiento [8].

Así las cosas, uno de los pasos que en nuestra experiencia constituye un mayor reto inicial para la puesta en marcha de un programa es la selección, diseño e implementación de escenarios de simulación. Los instructores han respondido a este reto convirtiendo la simulación en 'realista' con creatividad e innovación. Pero para que las simulaciones resulten a la vez atrayentes y efectivas para los participantes desde el punto de vista clínico, se deben sustentar sobre una base metodológica sólida. Esta necesidad contrasta con una escasez de guías estandarizadas y una referencia a las mejores prácticas para conducir la elaboración de escenarios clínicos de simulación. Constituye en la actualidad un reto y una oportunidad para los educadores la creación de un nuevo paradigma educativo que dé respuesta a la necesidad del alumno de una técnica docente interactiva, que estimule el juicio clínico y un pensamiento crítico transferibles a la práctica clínica [9].

El objetivo de este artículo es identificar y estandarizar las mejores prácticas para diseñar e implementar un escenario de simulación clínica. Se proporciona una guía para su preparación con fundamento metodológico en base al análisis de la experiencia adquirida en las actividades de entrenamiento para anestesiólogos desarrolladas en el Hospital Virtual Valdecilla. Se provee la información necesaria sobre todos los componentes, con aplicabilidad para entrenar una amplia variedad de competencias clínicas, para diferente experiencia de los participantes, y para un uso flexible de diferentes tipos de simuladores, incluyendo actores y maniquíes de distintos niveles de fidelidad. Las aplicaciones prácticas se dirigen tanto para aquellos instructores que están en las primeras fases de implementación de la simulación, como para quienes quieren expandir una aplicación clásica médica o quirúrgica.

\section{Materiales y métodos}

El análisis del proceso de diseño de un escenario clínico se realizó sobre los cursos de simulación realizados para la especialidad de Anestesiología y Reanimación en el Hospital Virtual Valdecilla durante el período septiembre 2008-junio 2011.

Se identificaron y estandarizaron las fases seguidas para el diseño, puesta en marcha y posterior refinamiento de cada escenario, así como los criterios utilizados para la toma de decisiones en cada una de ellas. A continuación se detallan siguiendo una secuencia de orden temporal.

\section{Estudio de las necesidades de entrenamiento}

Se exploró el tipo de participantes y sus necesidades de aprendizaje, teniendo en cuenta sus experiencias clínicas y las del resto del equipo, así como las observaciones de los responsables de sus áreas de trabajo. Una vez identificados los objetivos generales se determinó si la simulación podría ser más eficaz que otros métodos tradicionales de formación (sesiones clínicas, discusión de casos, rotaciones por el hospital), siguiendo los 'criterios para la selección de competencias susceptibles de entrenamiento mediante simulación clínica, descritos previamente [8].

\section{Definición de los objetivos específicos de aprendizaje}

Se consideraron, siempre desde la perspectiva del participante y teniendo en cuenta su nivel de formación, las habilidades que serían capaces de conseguir al final de su experiencia de simulación.

Se aplicó un enfoque jerárquico, es decir, asumiendo que el aprendizaje a niveles superiores depende de la adquisición del conocimiento y habilidades de ciertos niveles preliminares. Al mismo tiempo, se consideró un enfoque global del proceso educativo, promoviendo una forma de educación con un horizonte holístico. Para perseguir este doble objetivo, se siguió una taxonomía educativa modificada de Bloom [10]. En ella se incluyen, además de los conocimientos y su comprensión (p. ej.. dosis de los relajantes musculares), las habilidades manipulativas, manuales o físicas (dominio psicomotor; p. ej., técnica de intubación endotraqueal), el modo de procesar la información y las habilidades mentales (dominio cognitivo, como habilidades para resolver problemas; p. ej., el tipo de técnica no invasiva a elegir cuando falla la intubación), las actitudes y sentimientos ante la situación clínica (dominio afectivo; p. ej., reacción ante el estrés y modos de control de éste), las habilidades de comportamiento (habilidades interpersonales y trabajo en equipo; p. ej., comunicación y liderazgo) y los factores dependientes del sistema organizativo, sobre todo cuando el entrenamiento tiene un enfoque interdisciplinar.

\section{Agenda y programación del curso (logística)}

Una vez definidos los objetivos, en un siguiente paso se seleccionó el flujo de los objetivos de aprendizaje y las estrategias educacionales particulares para 
cada actividad (p. ej., entrenamiento en la toma de decisiones en pacientes con vía aérea difícil). Se determinó el número de estaciones a realizar, su duración y los horarios para que el participante alcanzase los objetivos planteados. Se identificaron la formación necesaria de los instructores y el número requerido, así como el número de actores y la necesidad o no de un técnico de simulación para preparar los escenarios y manejar los simuladores.

\section{Diseño de los escenarios}

Se creó una guía con todos los pasos considerados necesarios para el diseño de escenarios de simulación con los apartados que aparecen en la tabla I.

Para el desarrollo de los escenarios se dispuso de dos salas de simulación de alto realismo, dos salas de control, dos salas para talleres, tres salas para el entrenamiento de habilidades técnicas invasivas y dos salas para el análisis de las actuaciones. Asimismo se dispuso de un sistema de grabación audiovisual.

\section{Guía para el análisis}

Con propósito formativo, no evaluador (sumativo), para cada escenario se elaboró un listado con los objetivos específicos de aprendizaje planteados. La técnica docente para la reflexión y análisis de la experiencia vivida por los participantes fue la descrita por el Center for Medical Simulation (Cambridge, EE. UU.) [11].

\section{Refinamiento de los escenarios y evaluación del curso}

Al final de cada curso se realizaron encuestas escritas a los participantes para evaluar el grado de adecuación de la actividad a los objetivos docentes, incluyendo el realismo de los escenarios, su relevancia clínica y la calidad de los análisis. También se solicitó información a los responsables de las unidades.

\section{Presupuesto}

Cada actividad se presupuestó para facilitar la financiación de cada proyecto y hacerlo sostenible en el tiempo.

\section{Acreditación}

Se realizó de acuerdo con los requisitos, procedimiento y criterios establecidos por la Comisión de Formación Continuada, para tener efectos en todo el territorio nacional. Se consideran actividades de formación continuada acreditables aquellas activi-
Tabla I. Guía para el diseño de escenarios de simulación.

Objetivos docentes

Selección de referencias bibliográficas

Elementos necesarios para la preparación del escenario

Características de la sala de simulación

Tipo de simulador necesario y elementos para su caracterización

Material auxiliar

Historia clínica

Montaje del escenario

Descripción narrativa breve del escenario para todos

los participantes

Personal implicado en el escenario: actores y participantes

Resumen de los puntos clave del escenario para el personal de simulación

Guión del escenario (Tabla IV)

Parámetros para el simulador de pacientes

Lista de verificación previa al escenario

Guía para el análisis y herramientas de evaluación formativa

dades de enseñanza-aprendizaje que no están calificadas como formación reglada, y formación de grado o posgrado y especialidad, y que tengan como fin aumentar, mantener y mejorar la competencia profesional de los profesionales sanitarios.

\section{Resultados}

Se revisaron un total de $104(62+42)$ escenarios correspondientes a 14 cursos de formación continuada de médicos especialistas, y 13 realizados para el programa de integración de la simulación clínica en el programa formativo de los residentes de anestesiología y reanimación (Tablas II y III).

\section{Estudio de las necesidades de entrenamiento}

En el caso de los residentes de anestesia se creó un grupo de trabajo para identificar las competencias a entrenar, para lo cual se definieron unos criterios de selección de dichas competencias. Se eligieron para cada año de residencia aquellas que más pudieran beneficiarse de un aprendizaje basado en la propia 
Tabla II. Actividades de entrenamiento de especialistas de anestesiología y reanimación basadas en simulación clínica.

\begin{tabular}{|c|c|c|c|c|c|c|c|c|c|}
\hline & \multicolumn{3}{|c|}{ Participantes } & \multirow{2}{*}{$\begin{array}{l}\text { Sesión } \\
\text { teórica }\end{array}$} & \multicolumn{4}{|c|}{ Tipo de entrenamiento } & \multirow{2}{*}{$\begin{array}{c}\text { N.. o de } \\
\text { escenarios }\end{array}$} \\
\hline & Residentes & Especialistas & Equipo & & $\begin{array}{l}\text { Taller de } \\
\text { habilidades }\end{array}$ & $\begin{array}{l}\text { Toma de } \\
\text { decisiones }\end{array}$ & $\begin{array}{l}\text { Trabajo } \\
\text { en equipo }\end{array}$ & $\begin{array}{l}\text { Factores } \\
\text { del sistema }\end{array}$ & \\
\hline $\begin{array}{l}\text { Actualización en reanimación cardiopulmonar } \\
\text { en el paciente quirúrgico }\end{array}$ & $x$ & $x$ & & $x$ & $x$ & $x$ & $x$ & & 3 \\
\hline $\begin{array}{l}\text { Actualización en ventilación mecánica } \\
\text { para anestesiólogos }\end{array}$ & $x$ & $x$ & & $x$ & $x$ & $x$ & & & 3 \\
\hline Analgosedación para el paciente crítico & $x$ & $x$ & & $x$ & & $x$ & & & 5 \\
\hline Curso para instructores en simulación clínica & $x$ & $x$ & $x$ & $x$ & $x$ & $x$ & $x$ & $x$ & 10 \\
\hline $\begin{array}{l}\text { Decisiones diagnósticas y terapéuticas } \\
\text { en el paciente politraumático }\end{array}$ & $x$ & $x$ & & $x$ & & $x$ & $x$ & & 5 \\
\hline Manejo avanzado de la vía aérea con broncofibroscopio & $x$ & $x$ & $x$ & & $x$ & $x$ & $x$ & & 3 \\
\hline Manejo de la vía aérea difícil & $x$ & $x$ & $x$ & $x$ & $x$ & $x$ & $x$ & & 3 \\
\hline Retos comunicacionales en situaciones difíciles & $x$ & $x$ & & & & $x$ & & & 4 \\
\hline Patología obstétrica grave: abordaje interdisciplinar & $x$ & $x$ & $x$ & & & $x$ & $x$ & & 3 \\
\hline Reanimación neonatal & $x$ & $x$ & $x$ & & $x$ & $x$ & $\times$ & & 3 \\
\hline Toma de decisiones en analgesia perioperatoria & $x$ & $x$ & & $x$ & & $x$ & & $x$ & 6 \\
\hline
\end{tabular}

experiencia en ambientes simulados y que puedan integrarse con el currículo tradicional, sistematizándose en forma de actividades docentes [8].

En el caso de los especialistas, se trabajó en coordinación con la Jefatura del Servicio para identificar objetivos relacionados con la introducción de nuevas técnicas y tecnología, la actualización de protocolos en patologías prevalentes o el entrenamiento en situaciones raras pero con elevado riesgo de complicaciones.

\section{Definición de los objetivos específicos de aprendizaje}

Para cada escenario se eligió un número limitado de objetivos (tres o cuatro), estableciéndose una mezcla entre la toma de decisiones clínicas $(100 \%$ de los escenarios para los especialistas y residen- tes), las habilidades de comportamiento $(71,4 \%$ y $69,2 \%$, respectivamente), los factores relacionados con la organización (35,7\% y $30,7 \%$, respectivamente) y los factores relacionados con un enfoque interdisciplinar (50\% y $84,6 \%$, respectivamente).

\section{Agenda y programación del curso (logística)}

En cada actividad de los residentes se realizaron tres escenarios en una jornada de mañana, excepto en el curso de inmersión, en que se realizaron seis escenarios en tres jornadas de mañana. En siete de ellos se realizaron talleres para practicar las habilidades técnicas antes de las simulaciones clínicas. En los casos de abordaje de equipo, éste se componía de enfermeras de quirófano y residentes de cirugía general y de anestesia. En el caso de los espe- 
Tabla III. Actividades de simulación clínica en el programa formativo de los residentes de anestesiología y reanimación.

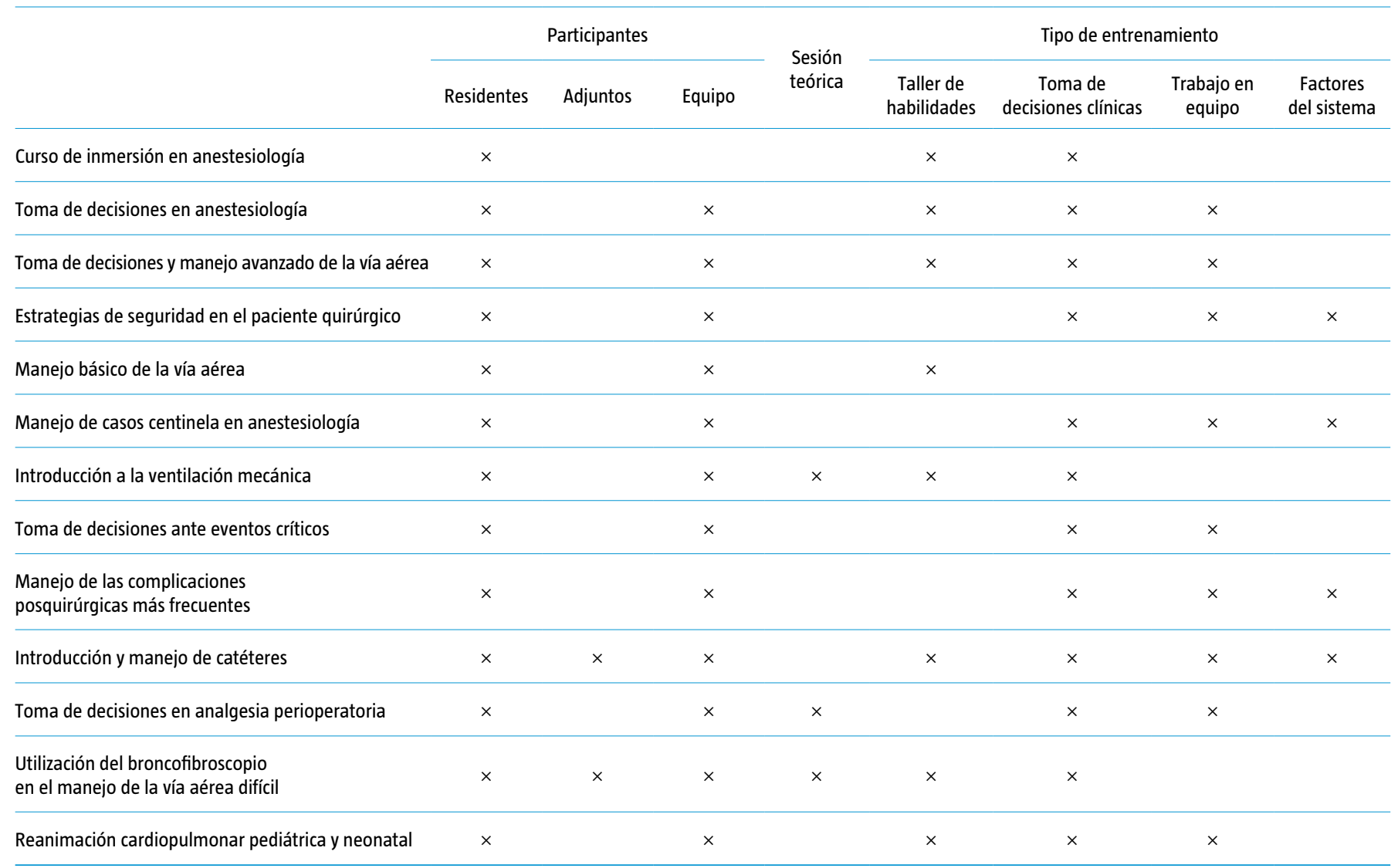

cialistas, el $71,4 \%$ de los cursos se realizaron en una jornada de mañana o tarde.

\section{Diseño de los escenarios}

Con el fin de crear experiencias de aprendizaje relevantes para los participantes se utilizaron tanto pacientes estandarizados como híbridos, así como maniquíes alta fidelidad, según los objetivos de cada caso clínico. En aquellas situaciones en que fue necesario realizar maniobras invasivas se utilizaron maniquíes, y en caso de que los objetivos fueran comunicacionales o relacionados con factores de la organización, se emplearon actores.

Para cada escenario se definió un guión con el fin de dirigir la evolución del caso clínico. El guión se elaboró siguiendo las cuatro fases que se reflejan en la tabla IV. Además, se definieron los signos vitales para introducir en el simulador estableciendo puntos de transición para cada fase. La evolución del paciente estuvo en consonancia con la práctica clínica mostrada por los participantes. También se preparó el material necesario para incorporar en la historia clínica del paciente (como analítica o pruebas de imagen).

\section{Guía para el análisis}

Todos los cursos estudiados tuvieron un objetivo formativo, no evaluativo, del personal sanitario. Todas las actividades se diseñaron con la misión de facilitar a los profesionales el desarrollo de habilidades prácticas y actitudes que potencien y estimulen la adquisición de las técnicas y capacidades que mejoren la calidad y seguridad de la asistencia, y de forma simultánea, faciliten la adaptación al cambio de la práctica clínica en función de la mejor evidencia clínica disponible en cada momento. En este sentido, para facilitar el análisis y reflexión sobre las actuaciones y disminuir la diferencia entre el rendimiento observado y el idealmente esperado, se utilizó la técnica descrita por el Center for Medical Simulation 
[11]. Los instructores responsables de los cursos tienen la certificación de haber completado el curso de instructores impartido por dicha institución.

\section{Presupuesto}

Para cada curso se realizó un cálculo de las necesidades de financiación, incluyendo la amortización y mantenimiento de los dispositivos de simulación, la participación de los instructores y los gastos generales de mantenimiento y desarrollo del centro imputados a cada actividad en particular. Todo ello con el objetivo de garantizar la sostenibilidad a largo plazo de los programas.

\section{Acreditación}

Los cursos para especialistas fueron acreditados por la Comisión de Formación Continuada del Sistema Nacional de Salud.

\section{Discusión}

Existe una evidencia creciente de que la simulación clínica promueve la integración de conocimientos y habilidades clínicas complejas [12], aumenta el grado de retención de lo aprendido cuando se compara con los métodos docentes tradicionales [13], es transferible al entorno clínico y mejora el rendimiento profesional $[14,15]$. Todo ello ha contribuido a que este tipo de entrenamiento se esté integrando de modo progresivo en los sistemas sanitarios modernos [16-18]. Sin embargo, debido a factores organizativos y de índole económico, la exposición de los profesionales sanitarios a las simulaciones es aún limitada [19]. Es por ello que las oportunidades de entrenamiento que se ofrezcan con esta técnica docente deben responder a problemas del mundo real, para que sean relevantes para el participante y faciliten la solución a los retos que se plantean en el día a día de las organizaciones sanitarias. En consecuencia, el diseño de escenarios clínicos se convierte en uno de los elementos fundamentales para que el empleo de la simulación facilite el desarrollo profesional y mejore la calidad de la asistencia de modo eficaz [20].

Este proceso de diseño comienza con el estudio de las necesidades de entrenamiento y la determinación de los objetivos. En nuestra experiencia, éste es el elemento clave para estructurar y comprender el proceso de aprendizaje. Se han definido diferentes dimensiones para guiar la formulación de objetivos significativos de aprendizaje:
- Adquisición de fundamentos teóricos.

- Aplicación de conocimientos y habilidades.

- Integración de distintas disciplinas utilizando pensamiento crítico y priorización en la toma de decisiones.

- Dimensión humana en la interacción con uno mismo y con el resto de miembros del equipo.

- Cuidado de paciente y su dimensión ética.

- Aprender a aprender de modo continuo durante toda la vida profesional [21].

Hemos utilizado una modificación de la taxonomía revisada de Bloom [10] con el objetivo de entrenar habilidades y aportar contenidos contextualizados con las tareas y actividades que realizan los profesionales. Este marco de trabajo permite observar el rendimiento al desempeñar tareas complejas que requieren habilidades de pensamiento superiores como la conceptualización, reflexión o pensamiento crítico. También posibilita estudiar la interacción con los miembros del equipo y dependiendo de las condiciones del sistema organizativo. Las variaciones del rendimiento clínico final pueden así atribuirse de modo individualizado al nivel de conocimiento previo, habilidad, motivación, personalidad, fatiga, condicionantes personales y ambientales.

La construcción de escenarios contextualizados con el paciente constituye un reto para los instructores de simulación. A menudo, las situaciones clínicas se plantean sin una comprensión del entorno social, ambiental y organizativo que rodea al paciente. El resultado puede ser un proceso de aprendizaje que no reproduce una situación similar a la real y que frustra las expectativas de los participantes. Las simulaciones se pueden planificar para presentar una amplia variedad de casos clínicos combinados con una gran diversidad de pacientes (en relación a sus necesidades, experiencias y diversidad social) y entornos de trabajo. La inclusión de estos factores y la inclusión de equipos multiprofesionales observada en un alto porcentaje de los cursos realizados facilita la comprensión de los procesos cognitivos de los participantes a la hora de adquirir conocimientos y habilidades, y desarrollar sus comportamientos, actitudes y creencias [22].

En nuestra experiencia, la participación de expertos clínicos en la materia objeto de entrenamiento, así como la de los responsables de las unidades implicadas, ayudó a individualizar las necesidades de entrenamiento.

La programación de las actividades se realizó en el centro de simulación porque permite un tiempo y espacio dedicados a la formación, y sirve a varios hospitales simultáneamente. Proporciona un espacio 
adecuado para disponer de equipos de simulación, grabación audiovisual y salas de análisis, y programar la agenda sin interferir con la actividad asistencial. En nuestra opinión, la participación de los profesionales en cursos de simulación en un centro de simulación facilita la comprensión en profundidad de las bases y alcance de esta técnica docente, y mejora el rendimiento de este tipo de actividad [23].

A la hora de determinar las características más relevantes de los escenarios surgen múltiples opciones, ya que éstos pueden cubrir una amplia variedad de dimensiones. Se pueden reproducir desde situaciones habituales a poco frecuentes, simples o complejas, con gran número o escasas tareas, que incluyan habilidades técnicas o solución de problemas. Además, dicha selección puede verse condicionada por el tipo de tecnología disponible, como simuladores de tareas, maniquíes robóticos, simuladores virtuales, pacientes estandarizados, pacientes híbridos (actores y simuladores de tareas juntos) o modelos animales [24]. En nuestro caso establecimos que el currículo guiase las características del escenario y la tecnología a emplear, y no al revés [25].

También es necesario establecer el grado de fidelidad a plantear para cada caso. En los primeros casos de un curso, los participantes a menudo tienen dificultades para orientarse en el escenario. Incluso aquellos que ya han tenido experiencias de simulación necesitan un tiempo para adaptarse al entorno y al simulador que están utilizando. Esta adaptación se dificulta si los signos del monitor no se corresponden con lo que dice el paciente o con los hallazgos de la exploración física. En este sentido se han descrito tres niveles de fidelidad a considerar: el realismo del entorno donde tiene lugar la simulación, el del equipo utilizado para el escenario, y el de la percepción del participante respecto a si la situación parece similar a la real [26]. Para familiarizar a los participantes con el ambiente y las potencialidades y limitaciones del maniquí, en algunos centros se enseña el entorno y el simulador antes de empezar los casos clínicos, con el fin de establecer las expectativas, para lo cual ayuda aclarar que no se está en el lugar habitual de trabajo. A veces se realizan casos de entrenamiento antes de empezar el curso. Es frecuente que existan dificultades para distinguir los artefactos producidos, que se interpretan como reales en el seno del escenario. Comúnmente se produce confusión respecto a los recursos -materiales y humanos- de que se dispone para manejar el caso.

Una vez decididas las características generales se elabora el guión, que viene determinado por la evolución del caso clínico. Se establecen los cambios
Tabla IV. Fases en la elaboración del guión de un escenario de simulación a .

Presentación de la situación clínica. Se provee un período inicial para facilitar:

La orientación con el entorno

Fase 1

El conocer al paciente, familiares y equipo clínico

La realización de la historia clínica

Los datos clínicos se hacen más relevantes. Es un tiempo para reconocer los problemas presentes y realizar:

Fase 2

Un enfoque diagnóstico

La toma de decisiones clínicas

La interacción con el equipo y familiares

Los problemas se hacen evidentes y el paciente evoluciona según la actuación clínica de los participantes:

Fase 3

Estabilización

Mejoría

Deterioro

Resolución. Es el momento para establecer el término del caso clínico:

Los objetivos se han presentado

Fase 4

Ha habido tiempo para tomar decisiones

Las intervenciones terapéuticas han podido hacer efecto

a Aspectos a tener en cuenta al elaborar un guión de un escenario: evitar excesivo detalle (hechos clínicos narrativos), anticipar árboles de decisión (diagrama de flujo con signos vitales), premiar la buena práctica de los participantes, prepararse para realizar cambios 'en directo', establecer puntos de transición para la evolución del caso, definir las acciones críticas del participante o resultados deseados, pensar en elementos de rescate.

previstos en la situación del paciente y sus constantes vitales, de modo que se puedan oír y ver durante el desarrollo del caso. Se pueden introducir elementos de distracción, conflicto o presión de producción dependiendo de los objetivos y las características de los participantes. En nuestro caso se construyeron escenarios desarrollados en tiempo real. Una forma de hacerlo es iniciar gradualmente la presentación del problema, permitiendo a los participantes que se involucren de manera progresiva en su diagnóstico y resolución. Si la presión de tiempo para realizar los escenarios es alta, un modo más eficiente es empezar con una alta carga de trabajo desde el principio o terminar el caso en medio del problema planteado. Se requiere un delicado balance en la dirección del escenario para crear un caso abierto a diferentes decisiones clínicas y, a la vez, facilitar que éste se mueva en una cierta dirección para que se logren los objetivos de aprendizaje [27]. La comunicación mediante sistemas de audio ina- 
lámbricos con los actores presentes en el escenario puede facilitar esta labor. En nuestra opinión, el diseño de escenario tiene tanto de ciencia como de arte, ya que el resultado final es determinado por una interacción dinámica en tiempo real entre el director del escenario y los participantes. Éstos pueden reaccionar de muy distintos modos, por lo que resulta útil anticipar y tener previstas posibles contingencias si la secuencia de acontecimientos no sigue el camino deseado [28].

Otro aspecto clave al diseñar los escenarios es determinar su validez para evocar los objetivos planteados. A menudo existen asunciones implícitas por parte de los instructores o limitaciones al desarrollo del caso, cuyas consecuencias a menudo no se evidencian hasta que se realiza el escenario en varias ocasiones. El objetivo es evaluar el impacto de la simulación en el participante, comprobar que los eventos, actores, simuladores, interacciones que se establecen y otras variables controlables evocan o replican los aspectos fundamentales de la realidad. Un escenario se probará válido si genera en el participante el modelo mental deseado y que coincida con los objetivos planteados. El grado de realismo requerido depende de este factor. Las claves para conseguirlo se deben identificar para que sea además reproducible en el tiempo. Estas características de reproducibilidad permiten estandarizar los escenarios clínicos y compartirlos entre diferentes centros de simulación para entrenar competencias específicas. Si se van a realizar evaluaciones durante el diseño también se debe pensar en cuáles van a ser los indicadores de las competencias a medir. El resultado de este proceso de validación será una información detallada de cómo se comporta este sistema bajo diferentes condiciones de participantes y situaciones [29]. En nuestra experiencia, el refinamiento constante de los escenarios permite identificar y seleccionar los elementos necesarios para acometer este proceso.

Por último, se necesita establecer el proceso de análisis de las actuaciones realizadas durante el escenario, definir con anticipación la técnica docente, con el objetivo de ayudar a los participantes en su desarrollo profesional [30]. El empleo del análisis con buen juicio, en el que se exploran las perspectivas del participante exponiendo de modo abierto los criterios del instructor, se ha mostrado superior a métodos que se basan en el juicio de las acciones o que se conducen mediante preguntas abiertas [11]. También resulta útil la combinación de guías para el análisis de la actuación clínica, las habilidades de comportamiento o de trabajo en equipo [31]. Existen múltiples escalas, unas miden resultados cuantitativos, y otras, globales, con o sin criterios de referencia [32].

En conclusión, el diseño de escenarios es un proceso iterativo y el empleo de una sistemática como la aquí descrita facilita su desarrollo. En nuestra experiencia, el análisis correcto de los objetivos docentes, y que éstos sean la guía para su diseño, constituye el factor individual más importante para el éxito de un programa. Como instructores de simulación estamos muy próximos a los casos que hemos ideado y, en ocasiones, resulta difícil anticipar el espectro de posibles reacciones que pueden provocar. Probar los escenarios con distintos tipos de participantes e identificar las características que los hacen válidos y reproducibles es el mejor modo de refinarlos.

\section{Bibliografía}

1. Morgan PJ, Cleave-Hogg D. Simulation technology in training students, residents and faculty. Curr Opin Anaesthesiol 2005; 18: 199-203.

2. Sancho R, Maestre JM, Del Moral I, Oest M, Carceller JM. Introducción segura de nueva tecnología en un servicio de anestesia. 29. ${ }^{\circ}$ Congreso de la Sociedad Española de Anestesiología, Reanimación y Terapéutica (SEDAR 09). Salamanca, 26-29 de mayo de 2009.

3. Del Moral I, Rabanal JM, Díaz de Terán JC. Simuladores en anestesia. Rev Esp Anestesiol Reanim 2001; 48: 423-33.

4. Fraser K, Peets A, Walker I, Tworek J, Paget M, Wright B, et al. The effect of simulator training on clinical skills acquisition, retention and transfer. Med Educ 2009; 43: 784-9.

5. Barsuk JH, McGaghie WC, Cohen ER, O'Leary KJ, Wayne DB. Simulation-based mastery learning reduces complications during central venous catheter insertion in a medical intensive care unit. Crit Care Med 2009; 37: 2697-701.

6. Gaba DM. The future vision of simulation in health care. Qual Saf Health Care 2004; 13 (Suppl 1): i2-10.

7. Nehring WN, Lashley FR. Current use and opinions regarding human patient simulators in nursing education: an international survey. Nurs Educ Perspect 2004; 25: 244-8.

8. Sancho R, Rábago JL, Maestre JM, Del Moral I, Carceller JM. Integración de la simulación clínica en el programa formativo de la especialidad de Anestesiología y Reanimación. Rev Esp Anestesiol Reanim 2010; 57: 656-63.

9. Anderson JM, Aylor ME, Leonard DT. Instructional design dogma: creating planned learning experiences in simulation. J Crit Care 2008; 23: 595-602.

10. Anderson LW, Krathwohl D, eds. A taxonomy for learning, teaching and assessing: a revision of Bloom's taxonomy of educational objectives. New York: Longman; 2001.

11. Rudolph J, Simon R, Dufresne RL, Raemer DB. There's no such thing as 'nonjudgmental' debriefing: a theory and method for debriefing with good judgment. Simul Healthc 2006; 1: 49-55.

12. Wisborg T, Brattebø G, Brinchmann-Hansen A, Einar P, Schrøder K. Effects of nationwide training of multiprofessional trauma teams in Norwegian hospitals. J Trauma 2008; 64: 1613-8.

13. Okuda Y, Bryson EO, DeMaria S Jr, Jacobson L, Quinones J, Shen B, et al. The utility of simulation in medical education: what is the evidence? Mt Sinai J Med 2009; 76: 330-43.

14. Fraser K, Wright B, Girard, L, Tworek, J, Paget, M, Welikovich L, et al. Simulation training improves diagnostic performance on a real patient with similar clinical findings. Chest 2011; 139: $376-81$. 
15. Kuduvallia PM, Parkerb CJR, Leuwerc M, Guha A. Retention and transferability of team resource management skills in anaesthetic emergencies: the long-term impact of a high-fidelity simulation-based course. Eur J Anaesthesiol 2009; 26: 17-22

16. Glavin RJ. Simulation: an agenda for the 21st century. Simul Healthc 2007; 2: 83-5.

17. Østergaard D. National medical simulation training program in Denmark. Crit Care Med 2004; 32: S58-60.

18. Sistac-Ballarín JM, Sáez-Fernández AS. II Jornadas de la Sección de Docencia y Formación de la Sociedad Española de Anestesiología y Reanimación. Rev Esp Anestesiol Reanim 2010; 57: 674-5.

19. Gaba D, Raemer D. The tide is turning: organizational structures to embed simulation in the fabric of healthcare. Simul Healthc 2007; 2: 1-3.

20. Childs JC, Sepples S. Clinical teaching by simulation: lessons learned from a complex patient care scenario. Nurs Educ Perspect 2006; 27: 154-8.

21. Fink LD. Creating significant learning experiences: an integrated approach to designing college courses. San Francisco, CA: Jossey-Bass; 2003

22. Mitchell R, Regan-Smith M, Fisher MA, Knox I, Lambert DR A new measure of the cognitive, metacognitive, and experiential aspects of residents' learning. Acad Med 2009; 84: 918-26.

23. Miller KK, Riley W, Davis S, Hansen HE. In situ simulation. A method of experiential learning to promote safety and team behavior. J Perinat Neonat Nurs 2008; 22: 105-13.

24. Cumin D, Merry AF. Simulators for use in anaesthesia. Anaesthesia 2007; 62: 151-62
25. Fort CW. Get a real-world education through simulation. Nursing 2009; 11: 47-9.

26. Campbell SH. Simulation scenarios for nurse educators: making it real. New York: Springer; 2009.

27. Lai F, Entin E, Dierks M, Raemer D, Simon D. Designing simulation-based training scenarios for emergency medical first responders. Proceedings of the Human Factors and Ergonomics Society 48th Annual Meeting 2004; 1670.

28. Dieckmann P, Lippert A, Glavin R, Rall M. When things do not go as expected: scenario life savers. Simul Healthc 2010; 5: 219-25.

29. Boulet JR, Murray D, Kras J, Woodhouse J, McAllister J, Ziv A. Reliability and validity of a simulation-based acute care skills assessment for medical students and residents. Anesthesiology 2003; 99: 1270-80.

30. Cheng A, Hunt EA, Donoghue A. EXPRESS -Examining Pediatric Resuscitation Education Using Simulation and Scripting. The birth of an international pediatric simulation research collaborative: from concept to reality. Simul Healthc 2011; 6: 34-41.

31. Hernández P, Odriozola JM, Maestre JM, López M Del Moral I, De Miguel JR. Entrenamiento de equipos interdisciplinares en urgencias obstétricas mediante simulación clínica. Prog Obstet Ginecol 2011; 54: 618-24.

32. Schartel SA, Metro DG. Evaluation: measuring performance, ensuring competence, achieving long-term excellence. Anesthesiology 2010; 112: 519-20. 